\title{
Smoking Influences the Yield of Dendritic Cells for Cancer Immunotherapy
}

Shimodaira $\mathbf{S}^{1 *}$, Higuchi $\mathbf{Y}^{1}$, Koya $\mathrm{T}^{1}$, Kobayashi $\mathrm{T}^{2}$, Yanagisawa $\mathbf{R}^{1}$, Hirabayashi $\mathrm{K}^{1}$, Ito $\mathrm{K}^{2,3}$, Koizumi $\mathrm{T}^{2}$, Maejima $\mathrm{S}^{4}$ and Udagawa $\mathbf{N}^{5}$

${ }^{1}$ Center for Advanced Cell Therapy, Shinshu University Hospital, Matsumoto, Japan

${ }^{2}$ Shinshu Cancer Center, Shinshu University Hospital, Matsumoto, Japan

${ }^{3}$ Department of Breast and Endocrine Surgery, Shinshu University Hospital, Matsumoto, Japan

${ }^{4}$ Internal Medicine, Matsumoto Dental University, Shiojiri, Japan

${ }^{5}$ Biochemistry, Matsumoto Dental University, Shiojiri, Japan

\begin{abstract}
Background: Dendritic cell (DC)-based vaccination is considered to be a potentially effective therapeutic strategy against advanced cancer. The aim of this study was to address the smoking history that might affect the preparation of DC vaccines in validated instructional manufacture.
\end{abstract}

Materials and Methods: Data on mature DCs generated from 102 sessions of leukapheresis performed on 92 patients with advanced cancer or sarcoma were retrospectively evaluated and compared in relation to the data between their smoking history and the generation of DCs from these patients. 61 patients with adenocarcinoma, including 7 with lung, 10 with breast, 8 with stomach, 12 with colorectal, and 23 with pancreatic adenocarcinoma were enrolled.

Results: The average yield of autologous DCs $\left(15.5 \pm 8.3 \times 10^{7}\right)$ was thought to be dependent on the number of monocytes $\left(124.2 \pm 74.1 \times 10^{7}\right)$ collected by leukapheresis. The average ratio of DCs/apheresed monocytes (DC/aM ratio) was lower in the smoker group $(11.1 \pm 7.2 \%)$ than that in the non-smoker group $(17.2 \pm 9.3 \%, p=0.001)$. The number of DCs and the DC/aM ratio were lower in the patients with gastric and pancreatic cancer than in those with adenocarcinoma of other sites.

Conclusions: As cancer therapy moves forward into the field of personalized therapies, we believe that these findings would be useful for individualized DC-based cancer immunotherapy.

Keywords: DC vaccination; Smoking; Cancer immunotherapy

Abbrevations: DC: Dendritic Cell; APC: Antigen-presenting Cell; WT1: Wilms Tumor 1; MUC1: Epithelial Membrane Antigen Mucin-1; HER2: Human Epidermal Growth Factor Receptor 2; CEA: Carcinoembryonic Antigen; PSA: Prostate-specific Antigen; GM-CSF: Granulocyte-macrophage Colony-stimulating Factor; GMP: Good Manufacturing Practice; OK-432: A Streptococcal Preparation; IL-4: Interleukin-4

\section{Introduction}

Despite the significant recent advances in the therapeutics for cancer, it still remains extremely difficult to treat advanced cancers with organ involvement and distant metastasis. Immunization against cancer still remains an underdeveloped field. Dendritic cell (DC)-based immunotherapy has been reported to be associated with few adverse reactions, but also to be of limited clinical success [1,2]. The ex vivo technique is being developed for DC-based cancer vaccination; however, it has been emphasized that a high quality of the manufactured DCs is needed for strong induction of the T cells against tumor antigens. DC-mediated vaccine studies have addressed correlations with tumor regression and/or improved survival, and also immune monitoring to define assays for protective lymphocytes [3-5]. Therefore, DC-mediated immunization against cancer with validated DC quality may potentially be expected to improve the protective effect of the vaccine against cancer as compared with the conventional approach.

A manufacturing technology for antigen-presenting cell (APC)based immunotherapy is being developed, with active DCs, the most potent APCs of the immune system, being under investigation for therapeutic vaccination against cancer. Immune DCs are generated from peripheral monocytes expressing tumor-specific antigens have made DCs applicable for active immunotherapy against cancers [6,7]. The most common approach to DC vaccination is the preparation of autologous mature monocyte-derived DCs ex vivo, which refers to large-scale in vitro generation of homogeneous, mature and functional DCs. A number of DC vaccination strategies have been used to induce an effective level of acquired immunity to achieve tumor regression [2,8-11]. Cancer vaccine therapies are principally attributed to the presence of tumor-associated antigens, such as WT1, MUC1, HER2, CEA, survivin, PSA, and others. According to the requirement of such antigens for therapeutic functions, immunogenecity, specificity, as well as oncogenecity, WT1 was found to be the most potent cancer-associated antigens fulfilling the immunological and clinical effectiveness needs among 75 vaccine peptides [12]. WT1 vaccination, an attractive target antigen for immunotherapy, has been shown to cause cancer regression without damaging normal tissues by inducing specific cytotoxic T lymphocytes [13,14]. To overcome the problem of the poor efficacy of cancer vaccination, WT1 peptide may be applied for DC-based immunotherapy with potentially strong therapeutic activity against cancers [15-19]. The yield of autologous DCs from monocytes obtained by leukapheresis varies from small to large, however, the factors directly or indirectly predictive of individual DC yields have not yet been clearly revealed. Large-scale preparations of DC vaccines with homogeneous, mature and functional profiles are a prerequisite for obtaining efficacious cancer immunotherapy $[4,5]$.

Cigarette smoking causes many types of cancers and death from cancers, including cancers of the lung, esophagus, larynx, mouth, throat, kidney, bladder, pancreas, stomach and cervix, as well as acute

*Corresponding author: Shigetaka Shimodaira, Center for Advanced Cell Therapy, Shinshu University Hospital, 3-1-1 Asahi, Matsumoto, Nagano 3908621, Japan, Tel: +81-263-37-3580; Fax: +81-263-37-3027; E-mail: shimodai@ shinshu-u.ac.jp

Received December 31, 2014; Accepted February 02, 2015; Published February 09, 2015

Citation: Shimodaira S, Higuchi Y, Koya T, Kobayashi T, Yanagisawa R, et al. (2015) Smoking Influences the Yield of Dendritic Cells for Cancer Immunotherapy. Pharmaceut Reg Affairs 4: 133. doi:10.4172/2167-7689.1000133

Copyright: (c) 2015 Shimodaira S, et al. This is an open-access article distributed under the terms of the Creative Commons Attribution License, which permits unrestricted use, distribution, and reproduction in any medium, provided the original author and source are credited. 
myeloid leukemia. An estimated $30 \%$ of the annual cancer deaths in the US attributed to smoking, and it has been demonstrated that quitting smoking can substantially reduce cancer risk, regardless of the age of the smoker [20]. WHO age-standardized prevalence of daily smoking among Japanese aged 15 years or more in 2011 was estimated to be $20 \%$ (31\% in males and $10 \%$ in females), with a tendency towards decrease, although the rate is still high as compared with that in the US or European countries [21].

The objective of this study was to address the cigarette smoking might affect a manufacture of the vaccine validated as good manufacturing practice (GMP) grade for clinical trial in immunotherapy against various types of cancer.

\section{Materials and Methods}

\section{Patients}

The Advanced Center for Cell Therapy in the Shinshu University Hospital (Matsumoto, Japan) investigated the efficacy of a vaccination strategy using WT1 -pulsed DCs for cancers and sarcomas. DC vaccination therapy at the Shinshu University Hospital was approved by the Ethics Committee of Shinshu University School of Medicine (approval number 1123, July $8^{\text {th }}, 2008$; and 1199, December 2, 2008). Cancer or sarcoma patients were enrolled for DC vaccination therapy after informed consent from each patient. $1-2 \times 10^{7}$ viable mature DCs prepared as bellow were administered by intradermal (i.d.) injection into the axillary and inguinal areas of the subjects. Tolerable 1 to $5 \mathrm{KE} /$ dose of OK-432 was injected together with the DCs either every 2 weeks or at intervals of the chemotherapy cycles, at least 5 to 7 sessions.

\section{Preparation of DCs}

$185 \mathrm{ml}$ of peripheral mononuclear cells were obtained by leukapheresis ( $400 \mathrm{ml} \times 13$ cycles) using a cell separator (AS TEC204, Fresenius, Germany) from 5,200 $\mathrm{ml}$ of the patient's blood. Adherent cells were cultured in AIM-V medium (Gibco, Gaithersburg, MD) containing granulocyte-macrophage colony stimulating factor (GMCSF, $50 \mathrm{ng} / \mathrm{ml}$; Gentaur, Brussels, Belgium), and IL-4 (50 ng/ml; R\&D Systems Inc., Minneapolis, $\mathrm{MN}$ ) to generate immature DCs in a $\mathrm{CO}_{2}$ incubator equipped with a Cell Processing Isolator $\left(\mathrm{H}_{2} \mathrm{O}_{2}\right.$-sterilizing system, SANYO Electric Co., Ltd., Osaka, Japan) [22] at the Cell Processing Center in Shinshu University Hospital. After 5 days of culture, these immature DCs were stimulated with OK-432 $(10 \mu \mathrm{g} /$ $\mathrm{ml}$; streptococcal preparation, Chugai Pharmaceutical Co, Ltd, Tokyo, Japan) and Prostaglandin E2 $(50 \mu \mathrm{g} / \mathrm{ml}$; Daiichi Fine Chemical Co. LTD., Toyama, Japan) for 24 hrs. The DCs were cryopreserved and kept until the day of administration. Generated DCs were pulsed with $100 \mu \mathrm{g} / \mathrm{ml}$ of WT1 peptide just before clinical use, with removing free peptides [23].

\section{Surface marker analysis of the yield DCs}

The phenotype of CD14, HLA-DR ${ }^{+}, \mathrm{HLA}-\mathrm{ABC}^{+}, \mathrm{CD}^{+} 0^{+}, \mathrm{CD}^{+} 3^{+}$, $\mathrm{CD}^{+} 6^{+}, \mathrm{CD}_{40}{ }^{+}$, and $\mathrm{CCR} 7^{+}$was taken as defining mature DCs [5]. To detect surface molecules expressed on the DCs yield in the fashion, flow cytometric analysis was performed using the FACS Calibur flow cytometer (BD Biosciences, San Jose, CA). The following antibodies were applied in viable DC populations excluded lymphocytes for cryopreserved samples: FITC-labeled anti-human CD14 (clone 61D3, eBiocience, San Diego, CA); CD40 (clone 5C3, eBiocience); CD80 (clone L307.4, BD Biosciences); HLA-ABC (clone W6/32, eBiocience); and CD3 (clone SK7, BD Biosciences); PE-labeled anti-human CD11c (clone B-ly6, BD Biosciences); CD83 (clone HB15e, eBiocience); CD86 (clone IT2.2, eBiocience); CD19 (clone 4G7, BD Biosciences), and HLA-DR (clone LN3, eBiocience).

\section{Statistical analysis}

Spearman's rank-order correlation was used to analyze the processes between apheresis of monocytes and the manufactured mature DCs. Differences of the peripheral blood, leukapheresed monocytes, number of DCs, and the DC ratio was determined by an unpaired $t$-test. Kruskal-Wallis (Wilcoxon) nonparametric test was applied to assess differences between subtypes of cancer, and the ratio for smoking factor in adenocarcinoma was evaluated by the chi-square test. Differences at $p<0.05$ were considered to be statistically significant. The analysis was performed on the software of SPSS, PASW Statistics 18 (ver. 18.0.0, SPSS Inc., Chicago, IL).

\section{Results}

\section{Apheresis for DC vaccination}

In a total 102 apheresis sessions in 92 patients with advanced cancer or sarcoma, the number of patients with pancreatic cancer was followed by those of breast, colorectal, stomach, and ovarian cancer, as shown in Table 1. Total number of apheresed monocytes was dependent on the peripheral monocyte count on the day of the apheresis, as shown in Figure $1(\mathrm{R}=0.696, p<0.0001, \mathrm{~N}=102)$. The mature DC product was statistically significantly dependent on the number of apheresed monocytes, because the yield of DCs varied from few to large, some factors other than apheresed monocytes might determine the qualitative/quantitative yield of the DC product Figure $2(\mathrm{R}=0.440$, $p<0.0001, \mathrm{~N}=102)$.

\section{Surface marker analysis of the yield DCs}

The number of DCs and viability before cryopreserved were 15.5 $\pm 8.3 \times 10^{7}, 95.7 \pm 4.6 \%$, respectively. Phenotype of the yield DCs with

\begin{tabular}{|c|c|}
\hline Cancer type & Total \\
\hline Brain tumor & 1 \\
\hline Salivary gland tumor & 2 \\
\hline Thyroid cancer & 2 \\
\hline Breast cancer & 10 \\
\hline Lung cancer (adenocarcinoma) & 7 \\
\hline Thymic cancer & 2 \\
\hline Gastric cancer & 8 \\
\hline Colorectal cancer & 12 \\
\hline Hepatic cancer & 1 \\
\hline Gall bladder cancer & 2 \\
\hline Bile duct cancer & 4 \\
\hline Pancreatic cancer & 23 \\
\hline Renal cell cancer & 1 \\
\hline Prostatic cancer & 1 \\
\hline Testicular cancer & 3 \\
\hline Ovarian cancer & 1 \\
\hline Uterine cancer & 8 \\
\hline Uterine sarcoma & 3 \\
\hline Melanoma & 1 \\
\hline Soft tissue sarcomas & 5 \\
\hline Malignant lymphoma & 3 \\
\hline
\end{tabular}

Table 1: Monocyte-based cancer immunotherapy: indication of cancer type. 
Citation: Shimodaira S, Higuchi Y, Koya T, Kobayashi T, Yanagisawa R, et al. (2015) Smoking Influences the Yield of Dendritic Cells for Cancer Immunotherapy. Pharmaceut Reg Affairs 4: 133. doi:10.4172/2167-7689.1000133

$\mathrm{CD} 11 \mathrm{c}^{+} \mathrm{CD} 14$ and $\mathrm{HLA}-\mathrm{ABC}^{+} \mathrm{DR}^{+}$were $95.6 \pm 9.3 \%$ (90\% ratio, $89.8 \%$ ), $97.5 \pm 5.7 \%$ (90\% ratio, $94.8 \%$ ), respectively (Figure 3 ). The $90 \%$ of the validated criteria met as higher as $5.1 \times 10^{7}$ cells with $91.9 \%$ of viability (Figure 3 ).

Mature DCs able to present tumor antigens to $\mathrm{CD} 8^{+} \mathrm{T}$-cells were confirmed by the surface phenotype of CD80, CD83, CD86, and HLAClass II. The yield DCs with mature phenotype strongly expressed CD11c, CD40, CD86, and HLA-Class I \& II, with few CD14 ${ }^{+}$monocyte markers. CD80 and CD83 positive DCs were varying among patients (Figure 4).

\section{Influencing monocyte-derived DC manufacture by smoking history}

The average age of the 92 patients undergoing the 102 sessions of apheresis was 56.8 years old, and the peripheral leukocyte and monocyte counts on the day of apheresis were $8,331 \pm 6,082 / \mu \mathrm{L}$ and $573 \pm 437 / \mu \mathrm{L}$, respectively. Average values in the apheresis product of the counts of mononuclear cells, harvested monocytes, and CD14 cells were $5.14 \pm 2.56 \times 10^{9}, 124.2 \pm 74.1 \times 10^{7}$, and $142.9 \pm 96.5 \times 10^{7}$, respectively. The mean number of mature DCs was $15.5 \pm 8.3 \times 10^{7}$, and the $\mathrm{DC} /$ monocyte-ratio and $\mathrm{DC} / \mathrm{CD} 14^{+}$cell ratio were $15.1 \pm 9.1 \%$ and $13.8 \pm 10.0 \%$, respectively (Table 2 ).

We identified that one of the factors correlated with the quantitative/ qualitative yields of autologous DC processing on the basis of the clinical records and hematological data during aphaeresis. A smoking history was identified as a negative factor influencing the DC yield. Cigarette

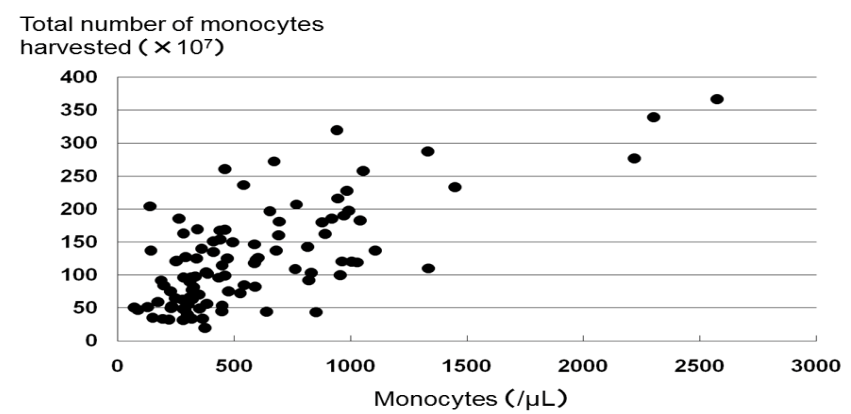

Correlation between peripheral blood monocyte counts and the number of monocytes harvested by apheresis: $\mathrm{R}=0.696, \mathrm{p}<0.0001, \mathrm{~N}=102$.

Figure 1: The yield of DCs dependent on the number of apheresed monocytes.

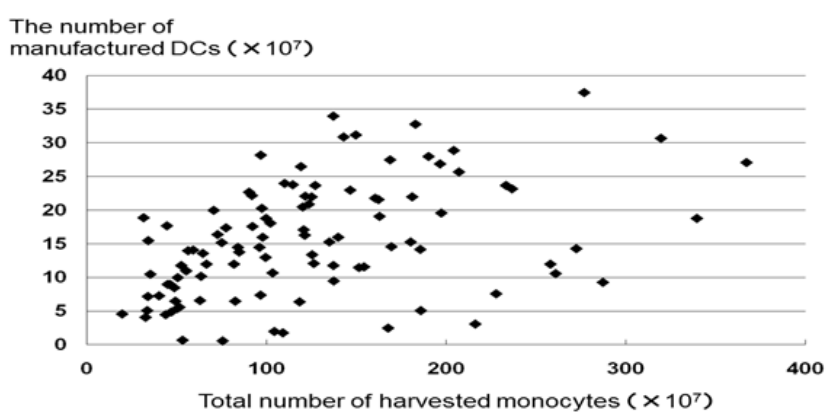

$\mathrm{R}=0.440, \mathrm{p}<0.0001, \mathrm{~N}=102$. The mean number of $\mathrm{DC}$ obtained from $124.2 \pm$ 74.1 apheresed monocytes was $15.4 \times 10^{7}$. The DC/apheresed monocyte ratio was calculated as $15.1 \pm 9.1 \%$.

Figure 2: The number of DCs dependent on the number of apheresed monocytes.

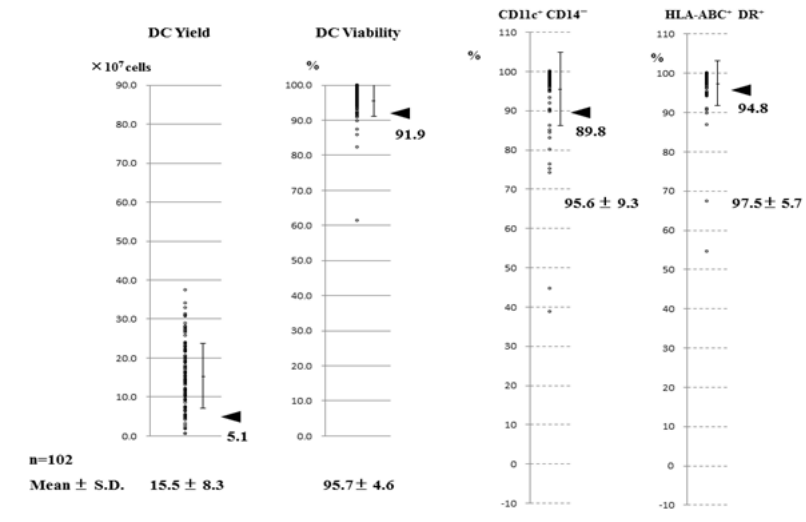

The number of DCs and viability before cryopreserved. Phenotype of the yield DCs with $C D 11 \mathrm{c}^{+} \mathrm{CD} 14^{-}$and $\mathrm{HLA}-\mathrm{ABC}^{+} \mathrm{DR}^{+}$populations representative of $D C$ markers are shown in the righter panels. The arrow heads indicate the validated criteria covering $90 \%$ of the products. Error bars on the dot plots are the mean $\pm \mathrm{SD}$.

Figure 3: Analysis of the yield of DCs.

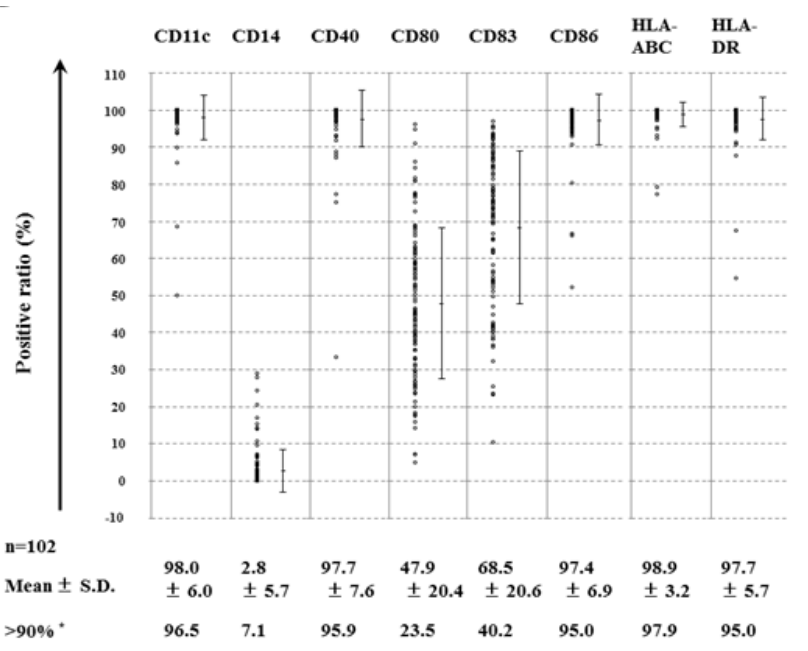

Error bars on the dot plots are the mean \pm SD. Mean positive ratios in the panel of CD11c, CD40, CD86, HLA-ABC, and HLA-DR display as over $90 \%$. *indicates minimum ratios to fulfill $90 \%$ of the yield DCs, except CD14, which marker require the lower criterion of the products.

Figure 4: Single color-flow cytometric analysis of the yield of DCs.

smokers were defined, based on their clinical records, as having a history of smoking over 10 cigarettes/day over 10 years (varying from 70 pieces for 16 years to 10 pieces for 50 years) prior to the diagnosis of cancer. There were no differences in the peripheral blood or apheresis bag data, or in the number of DCs between the smokers $\left(14.5 \pm 9.1 \times 10^{7}\right)$ and non-smokers $\left(16.0 \pm 7.9 \times 10^{7}\right)$; however, the percentage of DCs in the apheresed monocytes was lower in smokers $(11.1 \pm 7.2 \%)$ than that in the non-smokers $(17.2 \pm 9.3 \%, p=0.001)$, which was also confirmed by the $\mathrm{DC} / \mathrm{CD} 14^{+}$-ratio $(p=0.010)$. In the male/female ratio as $49 / 53$, the number of DCs in the females was higher $\left(17.2 \pm 8.6 \times 10^{7}\right)$ than that in the males $\left(13.6 \pm 7.7 \times 10^{7}, p=0.028\right)$; the percentage of DCs in the apheresed monocytes was also higher in the females $(17.7 \pm$ $10.0 \%)$ than that in the males $(12.3 \pm 7.1 \%$; $p=0.003)$. The ratio of the smokers/non-smokers was higher in the males (16/33) than that in the 
females (2/51), statistically significant determined by the chi-square test $(p<0.0001)$. Thus, the efficiency of autologous DC manufacture was low in patients who had a history of cigarette smoking, influencing the difference between genders.

\section{DC numbers among adenocarcinomas of different sites}

The DC yields in gastric cancer and pancreatic cancer were determined to be $10.3 \pm 6.3 \times 10^{7}$ and $13.7 \pm 6.9 \times 10^{7}$, respectively, lower than those in lung cancer $\left(20.7 \pm 9.0 \times 10^{7}\right)$ and colorectal cancer $(19.5$ $\pm 6.9 \times 10^{7}$ ) cancer patients, as shown in Table 3. The percentage of DCs in the monocytes was also shown to be statistically significantly lower in gastric cancer patients $(10.2 \pm 8.3 \%)$ and pancreatic cancer patients $(11.8 \pm 6.5 \%)$ as compared with that in breast cancer (17.7 $\pm 6.0 \%)$, colorectal cancer $(18.5 \pm 5.3 \%)$, and ovarian cancer $(18.4 \pm$ $6.8 \%)$ patients. The differences among the cancers were confirmed by the percentage of $\mathrm{CD}_{14}{ }^{+}$cells (Table 3 ). The distribution of gender, percentage of smokers and non-smokers, administration in patients with each of the cancers are shown in Table 3, indicating the absence of statistically significant differences among the cancers, except for breast cancer and ovarian cancer.

\section{Discussion}

As we move forward in the field of oncology to personalized therapies, feasible, well-tolerated and promising immunologic and multimodal therapies for various types of cancers or sarcomas would come to be expected [24]. The validation of quality standards for collection of sufficient amounts of autologous monocytes to achieve the expected level of cancer regression in DC-based immunotherapy is required. Reliable and reproducible collection of DCs would require throughout clinical applications and trials $[4,5,7]$.
We evaluated the data from 102 apheresis sessions performed in 92 patients with advanced cancer or sarcoma, of which pancreatic cancer accounted for about a quarter, as shown in (Table1). This is the first report to demonstrate that the quantity of autologous DCs that can be collected from cancer patients was influenced by individual smoking history. The number of DCs that could be collected ex vivo as well as the $\mathrm{DC} / \mathrm{aM}$ ratio differed among patients with different cancers. These parameters were especially low in pancreatic and stomach cancer patients as compared with the values in patients with other cancers such as lung, breast, colorectal and ovarian cancers, suggesting that the differing ex vivo differentiation of DCs among cancers might reflect the mechanisms underlying in vivo cancer immunity and cancer progression.

White blood cell counts are significantly higher in smokers, which is an independent risk factor for coronary heart disease, reviewed in the field of cardiology [25]. Smoking also stimulates monocyte adhesion, migration and activation, described as pathogenic mechanisms in the development atherosclerosis [26]; however, there has been no report of any correlation between cigarette smoking and the quantities of DCs that can be collected from patients. Smoking history is assumed as a confounding variable, associating with both leukocytosis and a relatively low efficiency of ex vivo DC collection, as shown in (Table2). These data might focus on how cigarette smoking might impact and potentiate the interference with the collection of DCs from patients ex vivo. Nicotine, one of the chemicals contained in cigarettes, is known to exert immunosuppressive effects on immune surveillance through impairment of DC functions [27]. Further studies are required to determine the mechanisms underlying DC immunity against cancer concerning cigarette smoking.

\begin{tabular}{|c|c|c|c|c|c|c|c|c|c|c|}
\hline & & & \multicolumn{2}{|c|}{ Peripheral blood } & \multicolumn{3}{|c|}{ Apheresis harvest } & \multicolumn{2}{c|}{ Dendritic Cells (DC) } \\
\hline & $\mathrm{N}$ & Age & $\begin{array}{c}\text { Leukocytes } \\
(/ \mu \mathrm{L})\end{array}$ & $\begin{array}{c}\text { Monocytes } \\
(/ \mu \mathrm{L})\end{array}$ & $\begin{array}{c}\text { Mononuclear } \\
\text { Cells }\left(\times 10^{9}\right)\end{array}$ & $\begin{array}{c}\text { Monocytes } \\
\left(\times 10^{7}\right)\end{array}$ & $\begin{array}{c}\text { CD14+ cells } \\
\left(\times 10^{7}\right)\end{array}$ & $\begin{array}{c}\text { Total number } \\
\left(\times 10^{7}\right)\end{array}$ & $\begin{array}{c}\text { Monocyte-ratio } \\
(\%)\end{array}$ & $\begin{array}{c}\text { CD14 } \pm \text { ratio } \\
(\%)\end{array}$ \\
\hline Total & 102 & 56.8 & $8,331 \pm 6,082$ & $573 \pm 437$ & $5.14 \pm 2.56$ & $124.2 \pm 74.1$ & $142.9 \pm 96.5$ & $15.5 \pm 8.3$ & $15.1 \pm 9.1$ & $13.8 \pm 10.0$ \\
\hline SEX & & & & & & & & & & \\
\hline Male & 49 & 55.9 & $8,108 \pm 5,689$ & $533 \pm 405$ & $5.22 \pm 2.69$ & $133.6 \pm 77.5$ & $155.4 \pm 103.3$ & $13.6 \pm 7.7$ & $12.3 \pm 7.1$ & $10.9 \pm 6.6$ \\
\hline Female & 53 & 57.6 & $8,537 \pm 6,471$ & $583 \pm 468$ & $4.79 \pm 2.40$ & $115.8 \pm 70.4$ & $130.8 \pm 89.0$ & $17.2 \pm 8.6$ & $17.7 \pm 10.0$ & $16.6 \pm 11.9$ \\
\hline & & & & & & & & $p=0.028$ & $p=0.003$ & $p=0.004$ \\
\hline $\begin{array}{l}\text { Smoking } \\
\text { History }\end{array}$ & & & & & & & & & \\
\hline Yes & 35 & 60.2 & $7,957 \pm 5,600$ & $641 \pm 453$ & $6.03 \pm 2.77$ & $154.8 \pm 80.2$ & $175.4 \pm 110.8$ & $14.5 \pm 9.1$ & $11.1 \pm 7.2$ & $10.3 \pm 7.1$ \\
\hline No & 67 & 55.0 & $8,526 \pm 6,351$ & $537 \pm 427$ & $4.68 \pm 2.33$ & $108.8 \pm 66.1$ & $125.3 \pm 83.7$ & $16.0 \pm 7.9$ & $17.2 \pm 9.3$ & $15.7 \pm 10.9$ \\
\hline & & & & & $p=0.011$ & $p=0.003$ & $p=0.013$ & & $p=0.001$ & $p=0.010$ \\
\hline
\end{tabular}

¥Smoking history: smoking over 10 rolls of cigarettes/day for over 10 years prior to the diagnosis of cancer; the ratio of the smokers/non-smokers was higher in the males $(16 / 33)$ than that in the females $(2 / 51)$.

Unpaired $t$-test, mean \pm S.D. $p<0.05$ indicates statistical significance.

Table 2: Factors predictive of the monocyte-derived DC yield MNC, mononuclear cells.

\begin{tabular}{|c|c|c|c|c|c|c|c|c|c|}
\hline & \multirow{3}{*}{$\mathbf{N}$} & \multirow{3}{*}{ Age } & \multirow{2}{*}{\multicolumn{2}{|c|}{ Sex }} & \multirow{2}{*}{\multicolumn{2}{|c|}{ Smoking }} & \multicolumn{3}{|c|}{ Dendritic Cells (DC) } \\
\hline & & & & & & & \multirow{2}{*}{$\begin{array}{c}\text { Total number } \\
\qquad\left(\times 10^{7}\right)\end{array}$} & \multirow{2}{*}{$\begin{array}{c}\text { Monocyte-ratio } \\
(\%)\end{array}$} & \multirow{2}{*}{$\begin{array}{c}\text { CD14 } \pm \text { ratio } \\
(\%)\end{array}$} \\
\hline & & & $\mathrm{M}$ & $\mathrm{F}$ & Yes & No & & & \\
\hline Lung cancer" & 7 & 57.7 & 5 & 2 & 5 & 2 & $20.7 \pm 9.0$ & $15.6 \pm 7.7$ & $14.6 \pm 8.0$ \\
\hline Breast cancer & 10 & 52.9 & 0 & 10 & 0 & 10 & $18.3 \pm 9.1$ & $17.7 \pm 6.0$ & $16.5 \pm 6.1$ \\
\hline Gastric cancer" & 8 & 62.3 & 5 & 3 & 4 & 4 & $10.3 \pm 6.3^{*}$ & $10.2 \pm 8.3^{*}$ & $9.8 \pm 9.0$ \\
\hline Pancreatic cancer ${ }^{\#}$ & 23 & 63.1 & 13 & 10 & 10 & 13 & $13.7 \pm 6.9^{*}$ & $11.8 \pm 6.5^{*}$ & $10.9 \pm 6.8^{*}$ \\
\hline Colorectal cancer ${ }^{\#}$ & 12 & 52.4 & 5 & 7 & 4 & 8 & $19.5 \pm 6.9$ & $18.5 \pm 5.3$ & $16.3 \pm 4.7$ \\
\hline Ovarian cancer & 8 & 52.9 & 0 & 8 & 0 & 8 & $15.4 \pm 7.3$ & $18.4 \pm 6.8$ & $17.6 \pm 6.7$ \\
\hline
\end{tabular}

\#Except for breast cancer and ovarian cancer, which are limited to female patients; the chi-square test indicated that there was no correlation between the cancer type and the gender or smoking history.

"Mann-Whitney's $U$ test was applied; mean \pm S.D. $p<0.05$ indicates statistical significance.

Table 3: Monocyte-DC processing from adenocarcinomas. 
Citation: Shimodaira S, Higuchi Y, Koya T, Kobayashi T, Yanagisawa R, et al. (2015) Smoking Influences the Yield of Dendritic Cells for Cancer Immunotherapy. Pharmaceut Reg Affairs 4: 133. doi:10.4172/2167-7689.1000133

\section{Conclusion}

Our study indicated that a smoking history affected the manufacture quantity of individualized DCs for vaccination in patients with various cancer types. We believe that these findings would contribute technically to the development of DC-based immunotherapy of cancers and sarcomas.

\section{Acknowledgments}

We also thank Ms. Kayo Horiuchi, Shinshu University Hospital, for her nursing support during the apheresis sessions.

\section{Disclosure of Interest}

All authors have no conflict of interest to declare, that could be perceived as prejudicing the impartiality of the research reported.

\section{References}

1. Rosenberg SA, Yang JC, Restifo NP (2004) Cancer immunotherapy: moving beyond current vaccines. Nat Med 10: 909-915.

2. Ridgway D (2003) The first 1000 dendritic cell vaccines. Cancer Invest 21 873-886.

3. Banchereau J, Steinman RM (1998) Dendritic cells and the control of immunity. Nature 392: 245-252.

4. Steinman RM, Banchereau J (2007) Taking dendritic cells into medicine. Nature 449: 419-426.

5. Figdor CG, de Vries IJ, Lesterhuis WJ, Melief CJ (2004) Dendritic cel immunotherapy: mapping the way. Nat Med 10: 475-480.

6. O'Neill DW, Adams S, Bhardwaj N (2004) Manipulating dendritic cell biology for the active immunotherapy of cancer. Blood 104: 2235-2246.

7. Nestle FO, Farkas A, Conrad C (2005) Dendritic-cell-based therapeutic vaccination against cancer. Curr Opin Immunol 17: 163-169.

8. Schlom J, Arlen PM, Gulley JL (2007) Cancer vaccines: moving beyond curren paradigms. Clin Cancer Res 13: 3776-3782.

9. Copier J, Ward S, Dalgleish A (2007) Cell based cancer vaccines: regulatory and commercial development. Vaccine 25 Suppl 2: 35-46.

10. Ramakrishnan R, Antonia S, Gabrilovich DI (2008) Combined modality immunotherapy and chemotherapy: a new perspective. Cancer Immunol Immunother 57: 1523-1529.

11. Baxevanis CN, Perez SA, Papamichail M (2009) Combinatorial treatments including vaccines, chemotherapy and monoclonal antibodies for cancer therapy. Cancer Immunol Immunother 58: 317-324

12. Cheever MA, Allison JP, Ferris AS, Finn OJ, Hastings BM, et al. (2009) The prioritization of cancer antigens: a national cancer institute pilot project for the acceleration of translational research. Clin Cancer Res15: 5323-5337.
13. Oka Y, Tsuboi A, Taguchi T, Osaki T, Kyo T, et al. (2004) Induction of WT1 (Wilms' tumor gene)-specific cytotoxic T lymphocytes by WT1 peptide vaccine and the resultant cancer regression. Proc Natl Acad Sci USA 101: 1388513890.

14. Oka Y, Tsuboi A, Oji Y, Kawase I, Sugiyama H (2008) WT1 peptide vaccine for the treatment of cancer. Curr Opin Immunol 20: 211-220.

15. Koido S, Homma S, Okamoto M, Takakura K, Mori M, et al. (2014) Treatment with chemotherapy and dendritic cells pulsed with multiple Wilms' tumor (WT1)-specific MHC class I/II-restricted epitopes for pancreatic cancer. Clin Cancer Res 20: 4228-4239.

16. Kobayashi M, Shimodaira S, Nagai K, Ogasawara M, Takahashi H, et al. (2014) Prognostic factors related to add-on dendritic cell vaccines on patients with inoperable pancreatic cancer receiving chemotherapy: a multicenter analysis. Cancer Immunol Immunother 63: 797-806.

17. Kobayashi M, Sakabe T, Abe H, Tanii M, Takahashi H, et al (2013) Dendritic cell-based immunotherapy targeting synthesized peptides for advanced biliary tract cancer. J Gastrointest Surg 17: 1609-1617.

18. Takahashi H, Okamoto M, Shimodaira S, Tsujitani S, Nagaya M, et al. (2013) Impact of dendritic cell vaccines pulsed with Wilms' tumour-1 peptide antigen on the survival of patients with advanced non-small cell lung cancers. Eur $J$ Cancer 49: 852-859.

9. Kimura Y, Tsukada J, Tomoda T, Takahashi H, Imai K, et al. (2012) Clinical and immunologic evaluation of dendritic cell-based immunotherapy in combination with gemcitabine and/or S-1 in patients with advanced pancreatic carcinoma. Pancreas 41: 195-205.

20. Surgeon General's Report (2014) The Health Consequences of Smoking-50 Years of Progress.

21. WHO report on the global tobacco epidemic (2013)

22. Kino-oka M, Taya M (2009) Recent developments in processing systems fo cell and tissue cultures toward therapeutic application. J Biosci Bioeng 108 267-276

23. Saito S, Yanagisawa R, Yoshikawa K, Higuchi Y, Koya T, et al. (2014) Safety and tolerability of allogeneic dendritic cell vaccination with induction of WT1specific T cells in a pediatric donor and pediatric patient with relapsed leukemia: A case report and review of the literature. Cytotherapy 0:1-6

24. Halama N, Zoernig I, Jaeger D (2010) Advanced malignant melanoma: immunologic and multimodal therapeutic strategies. J Oncol 2010: 689893

25. Madjid M, Awan I, Willerson JT, Casscells SW (2004) Leukocyte count and coronary heart disease: implications for risk assessment. J Am Coll Cardio 44:1945-1956.

26. Powell JT (1998) Vascular damage from smoking: disease mechanisms at the arterial wall. Vasc Med 3: 21-28.

27. Nouri-Shirazi M, Guinet E (2003) Evidence for the immunosuppressive role of nicotine on human dendritic cell functions. Immunology 109: 365-373. 